\title{
A dynamic reaction model of an inshore-offshore fishery with taxation as a control instrument
}

\author{
Jayjayanti Ray ${ }^{1}$, Tarun Pradhan ${ }^{2}$ \\ '(Department of Mathematics, Gokhale Memorial Girls' College, Kolkata, India) \\ ${ }^{2}$ (Department of Mathematics, Dinabandhu Andrews College, Kolkata, India)
}

\begin{abstract}
In this paper, we propose and analyse a mathematical model to study the dynamics of an inshoreoffshore fishery with taxation as a control instrument. The inshore area is considered as a breeding place having a fixed carrying capacity where fishing is strictly prohibited. Fishing is permitted only in the offshore area after imposing a tax per unit harvested biomass by the regulatory agency in order to control over exploitation. The steady states of the system are determined. The local and global stability of the interior equilibrium are discussed. Taking tax as a control variable, the optimal harvesting policy is formulated and solved by using Pontryagin's maximum principle [1]. The results are illustrated with the help of numerical example.
\end{abstract}

Keywords: Dynamic reaction, Inshore-offshore, Local and Global Stability, Optimal Harvesting, Pontryagin's maximum principle, Steady states

\section{Introduction}

In a dynamic model of an open-access fishery, the level of fishing effort expands or contracts according as the perceived rent (i.e. the net economic revenue to the fishermen) is positive or negative. A model reflecting this dynamic interaction between the perceived rent and the effort in fishery is called a dynamic reaction model.

In recent years, there is a spate of interest in bioeconomic analysis of exploitation of renewable resources like fisheries and forestries. Exploitation of natural resources has become a matter of concern throughout the world in view of problems caused by rapid depletion of these resources to meet the ever growing human needs. It has, therefore, become imperative to ensure scientific management of exploitation of biological resources. Gradual improvement in the technical efficiency of the fishing gear on vessels has radically changed the fishing scenario. Traditional fishing was mainly confined to the inshore coastlines. With the advent of sophisticated fishing instruments, offshore fishing has become more intensive than that in inshore.

Biologists and environmentalists are concerned that inshore fishing may cause over exploitation since many fish species like inshore area as breeding place. It is, therefore, necessary to formulate and analyse a mathematical model to study the dynamics of an inshore-offshore fishery and find out the techniques for controlling over-exploitation.

Regulation of exploitation of biological resources has become a problem of major concern nowadays in view of the dwindling resource stocks and the deteriorating environment. Exploitation of marine fisheries naturally involves the problems of law enforcement. The economics of law enforcement in marine fisheries was discussed by Sutinen and Andersen [2]. Several governing instruments are suggested for the choice of a regulatory control variable. These are imposition of taxes and licence fees, leasing of property rights, seasonal harvesting, direct control, etc. various issues associated with the choice of an optimal governing instrument and its enforcement in fishery were discussed by Andersen and Lee [3]. Optimal timing of harvest was adopted as a regulatory device by Kellog, Easely and Johnson [4] in their study of the North Carolina Bay Scallop Fishery. Imposition of a tax per unit biomass of the landed fish is a possible governing instrument in the regulation of fisheries. As described by Clark ([5], Art. 4.6, p.116), "Economists are particularly attracted to taxation, partly because of its flexibility and partly because many of the advantages of a competitive economic system can be better maintained under taxation than under other regulatory methods". However, it may be very difficult to impose tax in fishery due to some political and social problems. Nevertheless, the analysis of tax control gives a standard against which we may compare other controls. A single species fishery model using taxation as a control measure was first discussed by Clark ([5], Art. 4.6, p.116). Chaudhuri and Johnson [6] extended that model using a catch-rate function which was more realistic than that in [5]. Ganguly and Chaudhuri [7] made a capital theoretic study of a single species fishery with taxation as a control policy. Pradhan and Chaudhuri [8] developed a mathematical model for growth and exploitation of a schooling fish species, using a realistic catchrate function and imposing a tax per unit biomass of landed fish to control harvesting. Pradhan and Chaudhuri [9] also studied a fully dynamic reaction model of fishery consisting two competing fish species with taxation as a control instrument. Kar and Chattopadhyay [10] developed a dynamic reaction model of a prey-predator system where the selective harvesting of prey species is considered. Pradhan and Chaudhuri [11] proposed a 
model to study the selective harvesting in an inshore-offshore fishery on the basis of CPUE (catch per-uniteffort) hypotheses [5]. Srinivas et. al. [12] developed a prey-predator fishery model with stage structure in two patchy marine aquatic environment. They studied the dynamics of a fishery resource system with stage structure in an aquatic environment that consists of two zones - unreserved zone where fishing is permitted and reserved zone where fishing is prohibited.

The present paper deals with a dynamic reaction model in an inshore-offshore fishery, taking taxation as a control instrument. This model is developed by considering the inshore area as the breeding place where fishing is strictly prohibited and fishing is allowed only in the offshore area. The existence of possible steady states along with their local stability and the global stability of interior equilibrium are discussed. The optimal harvest policy is discussed using Pontryagin's maximum principle [1]. A numerical example is given as an illustration.

\section{Formulation Of The Problem}

Le us consider that at any time $t(>0), x_{1}(t)$ and $x_{2}(t)$ denote the respective biomasses of the inshore and offshore subpopulations of the same fishery. Let the fish subpopulation of the inshore area migrate into offshore area at a rate $\sigma_{1}\left(0<\sigma_{1}<1\right)$ and fish subpopulation of the offshore area migrate into inshore area at a rate $\sigma_{2}\left(0<\sigma_{2}<1\right)$. We also assume that the fish subpopulation of the inshore area obeys the logistic law of growth [13]. Under these considerations the fish subpopulations in the inshore and offshore areas obey the following system of differential equations :

$\frac{d x_{1}}{d t}=r x_{1}\left(1-\frac{x_{1}}{k}\right)-\sigma_{1} x_{1}+\sigma_{2} x_{2}$

$\frac{d x_{2}}{d t}=-s x_{2}+\sigma_{1} x_{1}-\sigma_{2} x_{2}$,

where $r, k, \sigma_{1}, \sigma_{2}, s$ are all positive constants.

Here $r=$ intrinsic growth rate of the inshore subpopulation,

$k=$ carrying capacity of the inshore area,

$\sigma_{1}=$ diffusion coefficient of inshore subpopulation,

$\sigma_{2}=$ diffusion coefficient of offshore subpopulation,

$s=$ natural mortality rate of the offshore subpopulation.

If $\sigma_{1}=0$, i.e. if there is no migration of the fish species from inshore area to offshore area then

$\frac{d x_{2}}{d t}=-\left(s+\sigma_{2}\right) x_{2}$

i.e. $x_{2}(t)=A_{0} e^{-\left(s+\sigma_{2}\right) t}, A_{0}$ is constant.

This implies offshore subpopulation exponentially decreases and tends to zero in future.

If $\sigma_{2}=0$, i.e. if there is no migration of the offshore subpopulation into inshore area, then

$\frac{d x_{1}}{d t}=r x_{1}\left(1-\frac{x_{1}}{k}\right)-\sigma_{1} x_{1}<0$ if $r<\sigma_{1}$ assuming $k \gg r$.

i.e. if the growth rate is less than the diffusion rate of the inshore subpopulation, the inshore subpopulation gradually decreases. So throughout our analysis we assume that $r>\sigma_{1}$ i.e. intrinsic growth rate of the inshore subpopulation is greater than its diffusion rate into offshore area.

We consider the inshore area as the breeding area of the population and so inshore area is the restricted area where fishing is strictly prohibited. Fishing is allowed only in the offshore area after imposing a tax implemented by the regulatory agency.

Let $E(t)$ be the harvesting effort for the offshore subpopulation and the regulatory agency imposes a tax $\tau(>0)$ per unit biomass of the harvested fish. The net economic revenue to the fisherman (perceived rent) is $\quad\left\{q(p-\tau) x_{2}-c\right\} E$, where $p=$ market price per unit biomass of the landed fish, $q=$ catchability coefficient of offshore subpopulation and $c=$ constant cost per unit of harvesting effort. $p-\tau>0$ i.e. $0<$ $\tau<p$ because it is obvious that market price $p$ per unit biomass of landed fish should be greater than the tax $\tau$ per unit biomass of landed fish.

Here we take $E(t)$ as a dynamic (time dependent) variable governed by the differential equation

$\frac{d E}{d t}=\lambda\left\{q(p-\tau) x_{2}-c\right\} E$,

where $\lambda$ is called the stiffness parameter measuring the effort and the perceived rent.

Therefore, we have the following system of differential equations:

$\left.\begin{array}{l}\frac{d x_{1}}{d t}=r x_{1}\left(1-\frac{x_{1}}{k}\right)-\sigma_{1} x_{1}+\sigma_{2} x_{2} \\ \frac{d x_{2}}{d t}=-s x_{2}+\sigma_{1} x_{1}-\sigma_{2} x_{2}-q E x_{2} \\ \frac{d E}{d t}=\lambda\left\{q(p-\tau) x_{2}-c\right\} E .\end{array}\right\}$ 


\section{The Steady States}

The steady states of the above system of equations are given by $P_{0}(0,0,0), P_{1}\left(\overline{x_{1}}, \overline{x_{2}}, 0\right)$ and $P_{2}\left(x_{1}^{*}, x_{2}^{*}, E^{*}\right)$

where

$$
\begin{aligned}
& \overline{x_{1}}=\frac{k}{r\left(s+\sigma_{2}\right)}\left\{m\left(s+\sigma_{2}\right)+\sigma_{1} \sigma_{2}\right\} \\
& \overline{x_{2}}=\frac{\sigma_{1} k}{r\left(s+\sigma_{2}\right)^{2}}\left\{m\left(s+\sigma_{2}\right)+\sigma_{1} \sigma_{2}\right\} \\
& x_{1}^{*}=\frac{k A m}{2 r} \\
& x_{2}^{*}=\frac{{ }_{c}}{(p-\tau) q} \\
& E^{*}=\frac{1}{q}\left\{\sigma_{1}\left(\frac{x_{1}^{*}}{x_{2}^{*}}\right)-\left(s+\sigma_{2}\right)\right\} \\
& m=r-\sigma_{1}>0 \text { by our assumption and } \\
& A=1+\sqrt{1+\frac{4 r c \sigma_{2}}{k(p-\tau) m^{2} q}}>2 \text { since } p>\tau>0 .
\end{aligned}
$$

Therefore, $\overline{x_{1}}>0, \overline{x_{2}}>0, x_{1}^{*}>0, x_{2}^{*}>0$.

But $\quad E^{*}>0$ iff $\left(\frac{x_{1}^{*}}{x_{2}^{*}}\right)>\frac{\left(s+\sigma_{2}\right)}{\sigma_{1}} \Rightarrow \tau<p-\frac{r c\left(s+\sigma_{2}\right)^{2}}{\sigma_{1} k q\left\{m\left(s+\sigma_{2}\right)+\sigma_{1} \sigma_{2}\right\}}$

Here $P_{0}(0,0,0)$ be the trivial steady state, $\left(\overline{x_{1}}, \overline{x_{2}}, 0\right)$ be the non-trivial steady state of the unexploited $(E=0)$ system and $P_{2}\left(x_{1}^{*}, x_{2}^{*}, E^{*}\right)$ be the unique non-trivial steady state of the exploited $(E \neq 0)$ system of $(1)$.

For the existence of the non-trivial steady state of the exploited system the regulatory agency has to impose a tax $\tau, 0<\tau<\tau_{\max }$ such that $\tau_{\max }=p-\frac{r c\left(s+\sigma_{2}\right)^{2}}{\sigma_{1} k q\left\{m\left(s+\sigma_{2}\right)+\sigma_{1} \sigma_{2}\right\}}$

When $\tau=\tau_{\max }$, then $p-\tau=\frac{r c\left(s+\sigma_{2}\right)^{2}}{\sigma_{1} k q\left\{m\left(s+\sigma_{2}\right)+\sigma_{1} \sigma_{2}\right\}}$ and $\quad E^{*}=0$.

Therefore, if the regulatory agency imposes maximum tax, then the system becomes unexploited i.e. fishing will be stopped. In that case,

$$
\begin{aligned}
x_{2}^{*} & =\frac{c}{\left(p-\tau_{\max }\right) q} \\
& =\frac{\sigma_{1} k}{r\left(s+\sigma_{2}\right)^{2}}\left\{m\left(s+\sigma_{2}\right)+\sigma_{1} \sigma_{2}\right\} \text { by }(9) \\
& =\frac{x_{2}}{x_{2}} \text { by }(3) .
\end{aligned}
$$

and $x_{1}^{*}=\frac{k m}{2 r}\left\{1+\sqrt{1+\frac{4 r c \sigma_{2}}{k\left(p-\tau_{\max }\right) m^{2} q}}\right\}$

$$
\begin{aligned}
& =\frac{k}{r\left(s+\sigma_{2}\right)}\left\{m\left(s+\sigma_{2}\right)+\sigma_{2}\right\} \text { by }(9) \\
& =\overline{x_{1}} \text {, by }(2) .
\end{aligned}
$$

It confirms that the non-trivial steady state $P_{2}\left(x_{1}^{*}, x_{2}^{*}, E^{*}\right)$ of the exploited $(E \neq 0)$ system becomes the non-trivial steady state $P_{1}\left(\overline{x_{1}}, \overline{x_{2}}, 0\right)$ of the unexploited $(E=0)$ system, when maximum tax is imposed by the agency.

\section{Local Stability}

The variational matrix of the system of equations (1) is

$$
V\left(x_{1}, x_{2}, E\right)=\left(\begin{array}{ccc}
r-\frac{2 r x_{1}}{k}-\sigma_{1} & \sigma_{2} & 0 \\
\sigma_{1} & -\left(s+\sigma_{2}+E q\right) & -q x_{2} \\
0 & \lambda q(p-\tau) E & \lambda\left\{(p-\tau) q x_{2}-c\right\}
\end{array}\right) .
$$

At $P_{0}(0,0,0), V(0,0,0)=\left(\begin{array}{ccc}r-\sigma_{1} & \sigma_{2} & 0 \\ \sigma_{1} & -\left(s+\sigma_{2}\right) & 0 \\ 0 & 0 & -\lambda c\end{array}\right)$.

One of the eigen values of $V(0,0,0)$ is $-\lambda c<0$ and the product of other two eigen values is $\left|\begin{array}{cc}r-\sigma_{1} & \sigma_{2} \\ \sigma_{1} & -\left(s+\sigma_{2}\right)\end{array}\right|=-\left(r-\sigma_{1}\right) s-r \sigma_{2}=-\left(m s+r \sigma_{2}\right)<0$, since $m=r-\sigma_{1}>0$.

Therefore, the variational matrix $V(0,0,0)$ has two negative eigen values and one positive eigen value. So $P_{0}(0,0,0)$ is an unstable steady state. 
At $P_{1}\left(\overline{x_{1}}, \overline{x_{2}}, 0\right), V\left(\overline{x_{1}}, \overline{x_{2}}, 0\right)=\left(\begin{array}{ccc}r-\frac{2 r \overline{x_{1}}}{k}-\sigma_{1} & \sigma_{2} & 0 \\ \sigma_{1} & -\left(s+\sigma_{2}\right) & -q \overline{x_{2}} \\ 0 & 0 & \lambda\left\{(p-\tau) q \overline{x_{2}}-c\right\}\end{array}\right)$

One of the eigen values of $V\left(\overline{x_{1}}, \overline{x_{2}}, 0\right)$ is $\lambda\left\{(p-\tau) q \overline{x_{2}}-c\right\}$.

This eigen value is positive or negative according as $p-\tau>$ or $<\frac{c}{q \overline{x_{2}}}$.

i.e. $\tau<$ or $>p-\frac{c r\left(s+\sigma_{2}\right)^{2}}{\sigma_{1} k q\left\{m\left(s+\sigma_{2}\right)+\sigma_{1} \sigma_{2}\right\}}$ by (3).

Sum of other two eigen values is trace of $\left(\begin{array}{cc}r-\frac{2 r \overline{x_{1}}}{k}-\sigma_{1} & \sigma_{2} \\ \sigma_{1} & -\left(s+\sigma_{2}\right)\end{array}\right)=-m-\frac{2 \sigma_{1} \sigma_{2}}{s+\sigma_{2}}-\left(s+\sigma_{2}\right)<0$.

Product of these two eigen values is $\left|\begin{array}{cc}r-\frac{2 r \overline{x_{1}}}{k}-\sigma_{1} & \sigma_{2} \\ \sigma_{1} & -\left(s+\sigma_{2}\right)\end{array}\right|=m\left(s+\sigma_{2}\right)>0$ by (2).

Therefore, these two eigen values are either real and negative or complex conjugates with negative real parts.

Therefore, the steady state $P_{1}\left(\overline{x_{1}}, \overline{x_{2}} 0\right)$ is unstable if $\tau<p-\frac{\operatorname{cr}\left(s+\sigma_{2}\right)^{2}}{\sigma_{1} k q\left\{m\left(s+\sigma_{2}\right)+\sigma_{1} \sigma_{2}\right\}}$ and asymptotically stable if $\tau>p-\frac{c r\left(s+\sigma_{2}\right)^{2}}{\sigma_{1} k q\left\{m\left(s+\sigma_{2}\right)+\sigma_{1} \sigma_{2}\right\}}$.

But $\tau<p-\frac{c r\left(s+\sigma_{2}\right)^{2}}{\sigma_{1} k q\left\{m\left(s+\sigma_{2}\right)+\sigma_{1} \sigma_{2}\right\}}$ is the necessary and sufficient condition for existence of the non-trivial steady state $P_{2}\left(x_{1}{ }^{*}, x_{2}{ }^{*}, E^{*}\right)$ for the exploited system. Thus we can state the following theorems:

Theorem-I: The non-trivial steady state of the exploited $(E \neq 0)$ system exists if and only if the non-trivial steady state of the unexploited $(E=0)$ system is unstable.

Theorem-II: The non-trivial steady state of unexploited $(E=0)$ system is asymptotically stable if and only if the non-trivial steady state of exploited $(E \neq 0)$ system does not exist.

These two theorems are also numerically verified in the section VII (Numerical Example).

$$
\text { At } \begin{aligned}
P_{2}\left(x_{1}^{*}, x_{2}^{*}, E^{*}\right), V\left(x_{1}^{*}, x_{2}^{*}, E^{*}\right) & =\left(\begin{array}{ccc}
r-\frac{2 r x_{1}^{*}}{k}-\sigma_{1} & \sigma_{2} & 0 \\
\sigma_{1} & -\left(s+\sigma_{2}+q E^{*}\right) & -q x_{2}^{*} \\
0 & \lambda q(p-\tau) E^{*} & \lambda\left\{(p-\tau) q x_{2}^{*}-c\right\}
\end{array}\right) \\
& =\left(\begin{array}{ccc}
-(A-1) m & \sigma_{2} & 0 \\
\sigma_{1} & -B & -q x_{2}^{*} \\
0 & \lambda q(p-\tau) E^{*} & 0
\end{array}\right)
\end{aligned}
$$

where $B=s+\sigma_{2}+q E^{*}=\sigma_{1} \frac{x_{1}^{*}}{x_{2}^{*}}>0$.

Characteristic equation of the matrix $V\left(x_{1}^{*}, x_{2}^{*}, E^{*}\right)$ is $y^{3}+m_{1} y^{2}+m_{2} y+m_{3}=0$

where $m_{1}=B+(A-1) m>0$ since $A>2, m>0$.

$$
\begin{aligned}
m_{2} & =m(A-1) B+\lambda q^{2}(p-\tau) E^{*} x_{2}^{*}-\sigma_{1} \sigma_{2} \\
& =\frac{m^{2} \sigma_{1} k q A(p-\tau)(A-1)}{2 r c}-\sigma_{1} \sigma_{2}+\lambda c q E^{*}
\end{aligned}
$$

and $m_{3}=\lambda q m c(A-1) E^{*}>0$, since $A>2$.

Since $A(A-1)>2$, so one of the sufficient condition for $m_{2}>0$ is $\tau<p-\frac{r c \sigma_{2}}{m^{2} k q}$

Now, $m_{1} m_{2}-m_{3}=\{B+m(A-1)\}\left\{m(A-1) B-\sigma_{1} \sigma_{2}\right\}+\lambda c q B E^{*}>0$ by (13).

Therefore, by Routh-Hurwitz stability criterion [14], condition (13) is the sufficient condition for local asymptotic stability of the non-trivial steady state $P_{2}\left(x_{1}^{*}, x_{2}^{*}, E^{*}\right)$ of the exploited system.

\section{Global Stability}

We have already proved that the system (1) has a unique non-trivial steady state $P_{2}\left(x_{1}^{*}, x_{2}^{*}, E^{*}\right)$ provided the condition (8) holds and this steady state is locally asymptotically stable with the sufficient condition (13).

Now, we shall prove that the non-trivial steady state $P_{2}\left(x_{1}^{*}, x_{2}^{*}, E^{*}\right)$ is globally asymptotically stable in the Lyapunov sense [15].

Let us consider the following Lyapunov function

$$
L\left(x_{1}, x_{2}, E\right)=\left\{x_{1}-x_{1}^{*}-x_{1}^{*} \ln \left(\frac{x_{1}}{x_{1}^{*}}\right)\right\}+L_{1}\left\{x_{2}-x_{2}^{*}-x_{2}^{*} \ln \left(\frac{x_{2}}{x_{2}^{*}}\right)\right\}+L_{2}\left\{E-E^{*}-E^{*} \ln \left(\frac{E}{E^{*}}\right)\right\}
$$

where $L_{1}, L_{2}$ are positive constant to be determined in the subsequent steps. 
Therefore, $\frac{d L}{d t}=\frac{x_{1}-x_{1}^{*}}{x_{1}} \frac{d x_{1}}{d t}+L_{1} \frac{x_{2}-x_{2}^{*}}{x_{2}} \frac{d x_{2}}{d t}+L_{2} \frac{E-E^{*}}{E} \frac{d E}{d t}$

$=\left(x_{1}-x_{1}^{*}\right)\left\{r-\frac{r x_{1}}{k}-\sigma_{1}+\sigma_{2} \frac{x_{2}}{x_{1}}\right\}+L_{1}\left(x_{2}-x_{2}^{*}\right)\left\{-s+\frac{\sigma_{1} x_{1}}{x_{2}}-\sigma_{2}-q E\right\}+L_{2} \lambda\left(E-E^{*}\right)\left\{(p-\tau) q x_{2}-c\right\}$,

by (1)

$$
\begin{aligned}
=\left(x_{1}-x_{1}^{*}\right)\{- & \left.\frac{r}{k}\left(x_{1}-x_{1}^{*}\right)+\sigma_{2}\left(\frac{x_{2}}{x_{1}}-\frac{x_{2}^{*}}{x_{1}^{*}}\right)\right\}+L_{1}\left(x_{2}-x_{2}^{*}\right)\left\{-q\left(E-E^{*}\right)+\sigma_{1}\left(\frac{x_{1}}{x_{2}}-\frac{x_{1}^{*}}{x_{2}^{*}}\right)\right\} \\
& +L_{2} \lambda q(p-\tau)\left(E-E^{*}\right)\left(x_{2}-x_{2}^{*}\right) \\
=-\frac{r}{k}\left(x_{1}-x_{1}^{*}\right)^{2} & +\frac{\sigma_{2}\left(x_{1}-x_{1}^{*}\right)\left(x_{2} x_{1}^{*}-x_{1} x_{2}^{*}\right)}{x_{1} x_{1}^{*}}-L_{1} q\left(x_{2}-x_{2}^{*}\right)\left(E-E^{*}\right)+L_{1} \frac{\sigma_{1}\left(x_{2}-x_{2}^{*}\right)\left(x_{1} x_{2}^{*}-x_{2} x_{1}^{*}\right)}{x_{2} x_{2}^{*}} \\
& +L_{2} \lambda q(p-\tau)\left(E-E^{*}\right)\left(x_{2}-x_{2}^{*}\right)
\end{aligned}
$$

Let $L_{1}=\frac{\sigma_{2} x_{2}^{*}}{\sigma_{1} x_{1}^{*}}>0$ and $L_{2}=\frac{\sigma_{2} x_{2}^{*}}{\sigma_{1} \lambda(p-\tau) x_{1}^{*}}>0$

Therefore, $\frac{d L}{d t}=-\frac{r}{k}\left(x_{1}-x_{1}^{*}\right)^{2}-\frac{\sigma_{2}}{x_{1}^{*}}\left\{\left(x_{2} x_{1}^{*}-x_{1} x_{2}^{*}\right)\left(\frac{x_{2}-x_{2}^{*}}{x_{2}}-\frac{x_{1}-x_{1}^{*}}{x_{1}}\right)\right\}$

$$
=-\frac{r}{k}\left(x_{1}-x_{1}^{*}\right)^{2}-\frac{\sigma_{2}}{x_{1} x_{2} x_{1}^{*}}\left(x_{2} x_{1}^{*}-x_{1} x_{2}^{*}\right)^{2}<0 .
$$

Hence, $\frac{d L}{d t}<0 \forall\left(x_{1}, x_{2}, E\right) \in R_{3}^{+} \backslash\left\{P_{2}\left(x_{1}^{*}, x_{2}^{*}, E^{*}\right)\right\}$ and $\frac{d L}{d t}=0$ at $P_{2}\left(x_{1}^{*}, x_{2}^{*}, E^{*}\right)$.

This shows that $\frac{d L}{d t}$ is negative definite in the region $R_{3}^{+} \backslash\left\{P_{2}\left(x_{1}^{*}, x_{2}^{*}, E^{*}\right)\right\}$ and hence by Liapunov's theorem on stability [15], it follows that the interior equilibrium $P_{2}\left(x_{1}^{*}, x_{2}^{*}, E^{*}\right)$ is globally asymptotically stable with respect to all solutions initiating in the interior of the positive quadrant.

\section{Optimal Harvest Policy}

In this section, we derive an optimal harvesting policy to maximize the total discounted net revenue from the harvesting biomass using the tax $\tau$ as a control parameter.

The net economic revenue $\pi\left(x_{1}, x_{2}, E, t\right)=$ net revenue of harvesting agency + net economic revenue of the

$$
\begin{aligned}
& \text { regulatory agency } \\
= & (p-\tau) q E x_{2}-c E+\tau q E x_{2}=\left(p q x_{2}-c\right) E
\end{aligned}
$$

The objective of the regulatory agency is to maximizing $J=\int_{0}^{\infty} e^{-\delta t}\left(p q x_{2}-c\right) E(t) d t$

where $\delta$ denotes the instantaneous annual rate of discount.

Our objective is to determine a tax $\tau$ to maximize $J$ subject to the state equations $\frac{d x_{1}}{d t}=\frac{d x_{2}}{d t}=\frac{d E}{d t}=0$ and the constraint $\tau_{\min }<\tau<\tau_{\max }$.

problem.

We apply Pontryagin Maximum Principle [1] to obtain the optimal equilibrium solution to this control

The Hamiltonian of this control problem is

$$
\begin{aligned}
H= & e^{-\delta t}\left(p q x_{2}-c\right) E+\lambda_{1}(t)\left\{r x_{1}\left(1-\frac{x_{1}}{k}\right)-\sigma_{1} x_{1}+\sigma_{2} x_{2}\right\}+\lambda_{2}(t)\left\{-s x_{2}+\sigma_{1} x_{1}-\sigma_{2} x_{2}-q E x_{2}\right\}+ \\
& \lambda_{3}(t)\left[\lambda\left\{(p-\tau) q x_{2}-c\right\} E(t)\right]
\end{aligned}
$$

where $\lambda_{1}(t), \lambda_{2}(t)$ and $\lambda_{3}(t)$ are adjoint variables.

Since $H$ is a linear function of $\tau$, the condition that the Hamiltonian $H$ be maximum for $\tau$ such that

$\tau_{\min }<\tau<\tau_{\max }$ is $\frac{\partial H}{\partial \tau}=0$.

Now, $\frac{\partial H}{\partial \tau}=0 \Rightarrow \lambda_{3}(t)=0$ by (15)

The adjoint equations are $\frac{d \lambda_{1}}{d t}=-\frac{\partial H}{\partial x_{1}}, \frac{d \lambda_{2}}{d t}=-\frac{\partial H}{\partial x_{2}}$ and $\frac{d \lambda_{3}}{d t}=-\frac{\partial H}{\partial E}$

Now, $\frac{d \lambda_{1}}{d t}=-\frac{\partial H}{\partial x_{1}} \Rightarrow \frac{d \lambda_{1}}{d t}=-\lambda_{1}\left\{r-\frac{2 r x_{1}}{k}-\sigma_{1}\right\}-\lambda_{2} \sigma_{1}$

$\frac{d \lambda_{2}}{d t}=-\frac{\partial H}{\partial x_{2}} \Rightarrow \frac{d \lambda_{2}}{d t}=-e^{-\delta t} p q E-\lambda_{1} \sigma_{2}+\lambda_{2}\left(s+\sigma_{2}+q E\right)$

and $\frac{d \lambda_{3}}{d t}=-\frac{\partial H}{\partial E} \Rightarrow \frac{d \lambda_{3}}{d t}=-e^{-\delta t}\left(p q x_{2}-c\right)+\lambda_{2} q x_{2}+\lambda_{3} \lambda\left\{(p-\tau) q x_{2}-c\right\}$

From (16) and (19) we have, $\lambda_{2}(t)=e^{-\delta t}\left(p-\frac{c}{q x_{2}}\right)$

Using the state equation $\frac{d x_{2}}{d t}=0$, from (20) we have $\frac{d \lambda_{2}}{d t}=-\delta e^{-\delta t}\left(p-\frac{c}{q x_{2}}\right)$

From (18) and (21), we have $\lambda_{1}(t)=\frac{e^{-\delta t}}{\sigma_{2}}\left\{p\left(s+\sigma_{2}\right)+\delta\left(p-\frac{c}{q x_{2}}\right)-\frac{c \sigma_{1} x_{1}}{q x_{2}^{2}}\right\}$

Again using the state equations $\frac{d x_{1}}{d t}=\frac{d x_{2}}{d t}=0$ and the equation (22), we have

$\frac{d \lambda_{1}}{d t}=-\frac{\delta e^{-\delta t}}{\sigma_{2}}\left\{p\left(s+\sigma_{2}\right)+\delta\left(p-\frac{c}{q x_{2}}\right)-\frac{c \sigma_{1} x_{1}}{q x_{2}^{2}}\right\}$ 
From (17) and (23), we have

$\left\{p\left(s+\sigma_{2}\right)+\delta p-\frac{c \delta}{q x_{2}}-\frac{c \sigma_{1} x_{1}}{q x_{2}^{2}}\right\}\left\{m-\delta-\frac{2 r x_{1}}{k}\right\}+\sigma_{1} \sigma_{2} p-\frac{\sigma_{1} \sigma_{2} c}{q x_{2}}=0, m=r-\sigma_{1}$

or, $P_{0}(m-\delta) x_{2}^{2}-\frac{2 P_{0} r}{k} x_{1} x_{2}^{2}-\frac{c \delta(m-\delta)}{q} x_{2}+\frac{2 c \delta r}{q k} x_{1} x_{2}-\frac{c \sigma_{1}(m-\delta)}{q} x_{1}+\frac{2 r c \sigma_{1}}{q k} x_{1}^{2}+\sigma_{1} \sigma_{2} p x_{2}^{2}-\frac{\sigma_{1} \sigma_{2} c}{q} x_{2}=0$,

where $P_{0}=p\left(s+\sigma_{2}\right)+\delta p>0$.

This gives the equation to the singular path.

From the state equation $\frac{d x_{1}}{d t}=0$, we have $x_{2}=\frac{1}{\sigma_{2}}\left(\frac{r x_{1}}{k}-m\right) x_{1}$.

From (24) and (25), we have, after a little algebraic calculation, the following equation:

$A_{0} x_{1}^{4}+A_{1} x_{1}^{3}+A_{2} x_{1}^{2}+A_{3} x+A_{4}=0$

where,

$$
\begin{aligned}
& A_{0}=\frac{2 P_{0} r^{3}}{k^{3} \sigma_{2}^{2}} \\
& A_{1}=\frac{r^{2}}{\sigma_{2}^{2} k^{2}}\left\{-(m-\delta) P_{0}-4 P_{0} m-\sigma_{1} \sigma_{2} p\right\} \\
& A_{2}=\frac{2 r}{k \sigma_{2}}\left\{\frac{P_{0} m^{2}}{\sigma_{2}}+\frac{m P_{0}(m-\delta)}{\sigma_{2}}-\frac{c r \delta}{q k}+\frac{\sigma_{1} \sigma_{2} p m}{\sigma_{2}}\right\} \\
& A_{3}=-\frac{P_{0}(m-\delta) m^{2}}{\sigma_{2}^{2}}+\frac{c \delta(m-\delta) r}{k q \sigma_{2}}+\frac{2 c \delta r m}{k q \sigma_{2}}-\frac{r c \sigma_{1}}{k q}-\frac{\sigma_{1} p m^{2}}{\sigma_{2}} \\
& A_{4}=-\frac{c \delta(m-\delta) m}{q \sigma_{2}}-\frac{c \delta \sigma_{1}}{q} .
\end{aligned}
$$

Let $x_{1 \delta}$ be the positive root (if exists) of the equation (26).

Then from (25) and the state equation $\frac{d x_{2}}{d t}=0$, we have

$x_{2 \delta}=\frac{1}{\sigma_{2}}\left(\frac{r x_{1 \delta}}{k}-m\right) x_{1 \delta}$,

and $\quad E_{\delta}=\frac{1}{q}\left\{\sigma_{1}\left(\frac{x_{1 \delta}}{x_{2 \delta}}\right)-\left(s+\sigma_{2}\right)\right\}$,

respectively.

Thus, the optimal equilibrium solution $\left(x_{1 \delta}, x_{2 \delta}, E_{\delta}\right)$ is obtained for a particular value of $\delta$.

In terms of these optimal equilibrium values, the optimal value of the tax $\tau$ is given by

$\tau_{\delta}=p-\frac{c}{q x_{2 \delta}}$.

\section{Numerical Example}

Let $r=5, k=1000, \sigma_{1}=0.5, \sigma_{2}=0.3, s=0.2, q=0.01, \lambda=1, p=10, c=48$ and $\delta=4$, in appropriate units.

As the regulatory agencies are always interested for the non-trivial steady states of exploited system, so using these parameter values the regulatory agencies have to choose the tax $\tau<5$ which satisfy the inequality (8), for existence of $P_{2}\left(x_{1}^{*}, x_{2}^{*}, E^{*}\right)$.

The results for different values of $\operatorname{tax} \tau$ are given in table-1.

Table 1: Steady states for different tax levels.

\begin{tabular}{|c|c|c|c|}
\hline $\boldsymbol{\tau}$ & $\boldsymbol{x}_{\mathbf{1}}^{*}$ & $\boldsymbol{x}_{\mathbf{2}}^{*}$ & $\boldsymbol{E}^{*}$ \\
\hline 0 & 931 & 480 & 47 \\
1 & 934.25 & 533.33 & 37.59 \\
2 & 938.36 & 600 & 28.20 \\
3 & 943.60 & 685.71 & 18.80 \\
4 & 950.50 & 800 & 9.41 \\
5 & $960\left(=\overline{x_{1}}\right)$ & $960\left(=\overline{x_{2}}\right)$ & 0.0 \\
6 & 973.93 & 1200 & -9.42 \\
\hline
\end{tabular}

From the above table, we see that when the tax increases, the equilibrium levels of subpopulations increases while the corresponding effort level decreases. This outcome is consistent with the social scenario that when any regulatory agency gradually increases the tax on fishing, the fishermen will be unwilling to go for fishing and so the harvesting effort gradually decreases and thereafter the equilibrium level of subpopulations gradually increases. 
Fig.1 is the graphical representation of the steady states of the system (1) in the domain $[0,5]$ of the tax $\tau$, where $\tau_{\min }=0$ and $\tau_{\max }=5$.

If the regulatory agency impose the maximum tax $\tau_{\max }=5$, then the effort level will be zero and then the steady state $P_{2}\left(x_{1}^{*}, x_{2}^{*}, E^{*}\right)$ of the exploited system becomes the steady state $P_{1}(960.960,0)$ of the unexploited system .

If the fishermen have to pay no tax i.e. $\tau=\tau_{\min }=0$, then the maximum equilibrium level of effort is $E_{\max }^{*}=47$ units and the minimum equilibrium level of subpopulations are $x_{1 \min }^{*}=931$ units and $x_{2 \max }^{*}=$ 480 units.

Since the regulatory agency has to choose the tax $\tau \in(0,5)$ for existence of the interior equilibrium $P_{2}\left(x_{1}^{*}, x_{2}^{*}, E^{*}\right)$, let the agency impose a tax $\tau=2$ units.

When $\tau=2$, then the non-trivial steady state of the exploited system is $P_{2}(938.36,600,28.20)$.

The characteristic equation of $V(938.36,600,28.20)$ is $y^{3}+5.666 y^{2}+18.27 y+66.108=0$, by (12).

Here $m_{1}=5.666>0, m_{2}=18.27>0, m_{3}=66.108>0$ and $m_{1} m_{2}-m_{3}=37.41>0$.

So by Routh-Hurwitz stability criterion the steady state $P_{2}(938.36,600,28.20)$ is locally asymptotically stable.

Using the above mentioned parameter values, $P_{1}(960,960,0)$ be the steady state of the unexploited system and for $\tau=2$, the eigen values of $V(960,960,0)$ are $-0.467619,-5.132381$ and 28.8 , by (11) and hence $P_{1}(960,960,0)$ is unstable.

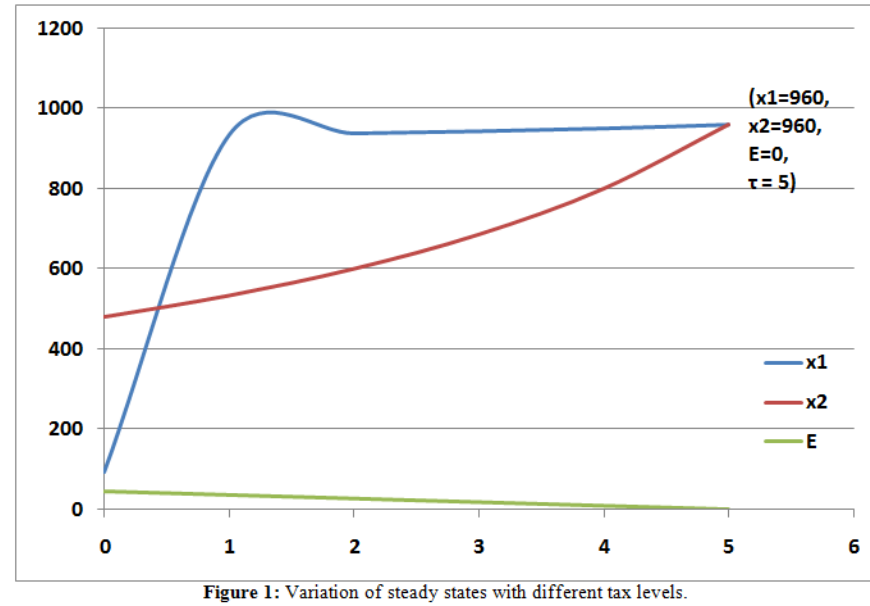

Figure 1: Variation of steady states with different tax levels.

Therefore, for $\tau=2$, the non-trivial steady state $P_{2}(938.36,600,28.20)$ of the exploited system exists and the steady state $P_{1}(960,960,0)$ of unexploited system is unstable. This verifies the theorem-I.

If the regulatory agency imposes the tax $\tau=6$ units, we see from the table that the steady state of exploited system does not exist but for $\tau=6$, the eigen values of the matrix $V\left(\overline{x_{1}}, \overline{x_{2}}, 0\right)$ at $P_{1}(960,960,0)$ are $-0.467619,-5.1323$ and -9.6 . So $P_{1}(960,960,0)$ is asymptotically stable. This verifies the theorem-II.

Using these parameter values equation (26) becomes

$0.000125 x_{1}^{4}-0.231667 x_{1}^{3}+110.05 x_{1}^{2}-2372 x_{1}-153600=0$.

The roots $x_{1 \delta}$ of this equation are $-27.528,53.495,893.745$ and 933.625.

But the first root is discarded because $x_{1 \delta}$ cannot be negative. For the other three positive roots, the corresponding values of $x_{2 \delta}$ are $-754.73,-93.173$ and 523.219 respectively.

Since $x_{2 \delta}$ cannot be negative, so the unique optimal value of $x_{2 \delta}$ is 523.219 corresponding to the optimal value 933.625 of $x_{1 \delta}$. For these optimal values of $x_{1 \delta}$ and $x_{2 \delta}$, we have from (28), $E_{\delta}=39.219$.

Therefore, $(933.625,523.219,39.219)$ is a unique optimal solution of the system.

The optimal tax is $\tau_{\delta}=0.826$, obtained from (29).

\section{Conclusion}

This paper deals with a problem of harvesting of an inshore-offshore fishery, considering inshore area as the breeding place where fishing is strictly prohibited. The most important feature of this model is that it assumes a fully dynamic interaction between the fishing effort and the perceived rent.

In this paper we give attention on the use of taxation as an optimal governing instrument to control the over exploitation of the offshore fish subpopulation. Ranges of taxation for existence of nontrivial steady state and the optimal equilibrium solution are discussed. 
The changes of steady states corresponding to the changes of taxation in the permissible range, is shown graphically by the numerical example .

\section{References}

[1] Pontryagin L.S., Boltyanskii V.S., Gamkrelidze R.V. and Mishchenko E.F., The Mathematical Theory of Optimal Process ( WileyInterscience, New York, 1962).

[2] Sutinen, J.G. and Andersen, P., The economics of fisheries law enforcement, Land Economics, 61(4), 1985, $387-397$.

[3] Anderson, L.G. and Lee, D.R., Optimal governing instrument, operation level and enforcement in natural resource regulation: the case of the fishery, American Journal of Agricultural Economics, 68, 1986, 678-690.

[4] Kellog R.L., Easley J.E. Jr. and Johnson T., Optimal timing of harvest for the North Carolina Bay Scallop Fishery, American Journal of Agricultural Economics, 71(1), 1988, 50-62.

[5] Clark C.W. Mathematical Bioeconomics: The Optimal Management of Renewable Resources ( John Wiley and Sons, New York, 1976).

[6] Chaudhuri K.S. and Johnson, T. , Bioeconomic dynamics of a fishery modeled as an S-system. Mathematical Biosciences, 99, 1990, 231-249.

[7] Ganguly S. and Chaudhuri K.S., Regulation of a single-species fishery by taxation. Ecological Modelling, 82, 1995, 51-60.

[8] Pradhan T. and Chaudhuri K.S., Bioeconomic harvesting of a schooling fish species: a dynamic reaction model. Korean Journal for Computational \& Applied Mathematics, 1, 1999, 127-141.

[9] Pradhan T. and Chaudhuri K.S. , A dynamic reaction model of a two species fishery with taxation as a control instrument: a capital theoretic analysis. Ecological Modelling, 121, 1999, 1-6.

[10] Kar T.K. and Chattopadhyay S.K., A dynamic reaction model of a prey-predator system with stage-structure for predator. Modern Applied Science, 4, 2010, 183-195.

[11] Pradhan T. and Chaudhuri K.S., Bioeconomic modeling of selective harvesting in an inshore-offshore fishery. Differential Equations and Dynamical Systems, 7, 1999, 305-320.

[12] Srinivas M. N., Srinivas M. A. S., Das K. and Gazi N.H., Prey predator fishery model with stage structure in two patchy marine aquatic environment. Applied Mathematics, 2, 2011, 1405-1416.

[13] Verhulst, P.F., Notice Sur la loi que la population suit dans son accroissment, Correspondence Mathematique et Physique, 10, $1838,113-121$.

[14] Goh B.S., Management and analysis of biological populations (Elsevier, Amsterdam, 1980).

[15] Salle J. La, Lefschetz, S. (1961). Stability by Liapunov's Direct Method with Application ( Academic Press, New York, 1961). 